This item was submitted to Loughborough's Research Repository by the author.

Items in Figshare are protected by copyright, with all rights reserved, unless otherwise indicated.

\title{
Dual screening, public service broadcasting, and political participation in eight Western democracies
}

PLEASE CITE THE PUBLISHED VERSION

https://doi.org/10.1177/1940161218779170

\section{PUBLISHER}

(C) the Authors. Published by SAGE Publications

\section{VERSION}

AM (Accepted Manuscript)

\section{PUBLISHER STATEMENT}

This work is made available according to the conditions of the Creative Commons Attribution-NonCommercialNoDerivatives 4.0 International (CC BY-NC-ND 4.0) licence. Full details of this licence are available at: https://creativecommons.org/licenses/by-nc-nd/4.0/

\section{LICENCE}

CC BY-NC-ND 4.0

\section{REPOSITORY RECORD}

Vaccari, Cristian, and Augusto Valeriani. 2018. "Dual Screening, Public Service Broadcasting, and Political Participation in Eight Western Democracies”. Loughborough University. https://hdl.handle.net/2134/33103. 


\section{Dual Screening, Public Service Broadcasting, and Political Participation in Eight Western Democracies}

Cristian Vaccari (Loughborough University)

Augusto Valeriani (University of Bologna)

Accepted for publication in the International Journal of Press/Politics on 4 May 2018

Abstract

We investigate the relationship between political dual screening - that is, watching political contents on television while reading and commenting on them on social media-and political participation across eight Western democracies: Denmark, Germany, Greece, Italy, Poland, Spain, United Kingdom, and United States. Based on custom built online surveys conducted between 2015 and 2016 on samples representative of the adult population with internet access in each country, we test hypotheses on both intra-country and cross-country direct and differential effects of political dual screening on various forms of offline and online political participation. We find a positive correlation between the frequency with which citizens dual screen political content and their overall levels of participation. Such correlation is stronger among respondents with lower levels of interest in politics, suggesting that dual screening has the potential to bridge participatory gaps between citizens who are more and less politically involved. The relationship between dual screening and participation is also significantly stronger in countries whose media systems feature the strongest Public Service Broadcasters. Our findings suggest that dual screening makes a positive contribution to democratic citizenship and political equality, and that it can also help public service media fulfill some of their key functions. 


\section{Introduction}

Political dual screening entails combining television and social media in attending to and engaging with political issues and events. This practice is increasingly popular in democratic regimes. Seventy-four percent of citizens across 26 democracies get their news on television and 51\% do so on social media at least weekly (Reuters Institute for the Study of Journalism 2016). These two channels are often combined in real time: for instance, $37 \%$ of those who followed the results of the 2016 United States Presidential elections did so simultaneously on television and online (Pew Research Center 2016). The integration of social media and television is apparent during high-profile live political events such as leaders' debates, where broadcasters lure viewers online (Chadwick 2017), campaigners strive to affect the public's and journalists' interpretations of the event (Kreiss 2016), and citizens learn about, discuss, and seek to shape others' interpretations (Chadwick et al. 2017).

In this article, we contribute to the increasing evidence that dual screening may play a relevant role in contemporary citizenship (Gil de Zúñiga, Garcia-Perdomo, and McGregor 2015), focusing on the relationship between dual screening political content and participation. Based on unique surveys of samples representative of adult internet users in eight Western democracies—Denmark, Germany, Greece, Italy, Poland, Spain, the United Kingdom, and the United States-we show that dual screening political content is positively and significantly correlated with political participation, measured across online and offline domains. We also demonstrate that such positive correlation is stronger among citizens with lower levels of interest in politics and in countries with strong Public Service Broadcasters.

\section{Literature Review, Hypotheses, and Research Questions}

Watching political programs on TV while simultaneously producing and consuming related content on social media has become popular across the world (Gil de Zúñiga and Liu 2017). So 
far, the literature on political dual screening has begun theorizing this emerging phenomenon, situating it among other practices of TV consumption, and studying its relationships with political engagement.

Vaccari, Chadwick and O'Loughlin define dual screening as a bundle of "lean back" and "lean forward" practices that involve simultaneous or alternate uses of television and social media to engage with mediated political content and events (Vaccari et al. 2015: 1044). Dual screening is a typical practice of hybrid media systems, where older and newer media logics are intertwined in individuals' experience of and engagement with politics (Chadwick 2017). Some studies of dual screening focus on the relationships between the logics of the two screens, finding that the contents, types, and intensity of citizens' activities on social media reflect concurrent TV performances (Shah et al. 2016; Giglietto and Selva 2014). However, the logics of the two screens are not necessarily always congruent. For one, political dual screeners frequently leverage social media to introduce information, topics and interpretations that deviate from what is happening on TV (Anstead and O'Loughlin 2011; Trilling 2015). Moreover, dual screeners often end up watching televised political events by accident, as a result of being exposed to relevant content while scrolling their social media timelines for other reasons (Vaccari et al. 2015).

Political dual screeners do not simply consume televised news but engage with political content at different levels of activity across older and newer platforms. Thus, the affordances and practices that constitute political dual screening, and their implications for citizens' participation, should be studied in their own right, rather than being treated as a simple extension of television watching or as yet another way of interacting with political messages on social media (Choi and Jung 2016).

The debate over the effects of television on political engagement and the quality of civic life is long-standing. Initially, many scholars argued that watching TV bred political 
apathy (Robinson 1976) and depleted social capital (Putnam 1995). Later studies, however, showed that consumption of specific TV genres, such as news and public affairs programs (Norris 2000), or of outlets offering substantial amounts of political content (De Vreese and Boomgaarden 2006), positively contributes to political knowledge and participation.

A similar debate has developed more recently on the role of social media for political information, discussion, and participation. Studies have shown that reading news, posting messages, and engaging in political discussions on social media are antecedents to political participation occurring both online and offline (Boulianne 2015; Cantijoch et al. 2014; Gil de Zúñiga et al. 2014; Yang and DeHart 2016).

Few studies so far have addressed the nexus between dual screening and political participation. Most of them (see Gil de Zúñiga and Liu 2017 for a review) concluded that dual screeners tend to participate in politics more than non-dual screeners. To identify the theoretical grounds that may explain the positive relationship between political dual screening and participation, Gil de Zúñiga, Garcia-Perdomo, and McGregor (2015, p. 794) propose a combination of the Communication Mediation Model (McLeod et al. 1999) and the Cognitive Mediation Model (Eveland 2004). Both frameworks emphasize the central role of interpersonal communication in enhancing elaboration and self-reflexivity around political information, in turn making it more likely that individuals will act upon such information. That such an interpersonal enhancement may occur is made more likely by the fact that key motivations for political dual screening are the desire to obtain additional information on the TV programs being watched and the satisfaction users get from discussing broadcasts with others (Gil de Zúñiga et al. 2015). By combining exposure to televised content and interpersonal communication about it, political dual screening thus "involves additional news consumption, discussion, and, at least sometimes, elaboration" (Gil de Zúñiga et al. 2015, p. 
795), which then results in higher probability to participate in politics as an outcome of such peculiar way of engaging with, discussing and interpreting political content.

Following a similar theoretical path, Barnidge, Gil de Zúñiga, and Diehl (2017) find that dual screening makes social media users more permeable to political persuasion. Barnidge and colleagues (2017) argue this is because, first, dual screeners acquire information and opinions related to the TV broadcast from other users in the very same moment they are themselves processing television content. This combination makes dual screening potentially more influential in orienting individuals' views than other types of digital political discussion. Secondly, social cues encountered on social media provide indications of the popularity of different contents and might make subjects more inclined to be influenced by dual screened messages that appear to be more popular (Barnidge et al. 2017).

Dual screening is thus a peculiar hybrid experience that can involve cognitions and actions taking place not only during the airing of a program but also after it (Auverset and Billings 2016). The studies mentioned above indicate that the distinctive features of dual screening may positively affect citizens' political participation. However, most of this research has focused on a single country-often the United States-and the sole comparative study that is currently available (Gil de Zúñiga and Liu 2017) only provides bivariate measures of association between dual screening and participation. We aim to place research in this field on a more solid empirical ground by providing multivariate assessments of the direct relationship between dual screening and participation across eight Western democracies. Thus, we test the following hypothesis:

H1: The frequency with which individuals dual screen political content is positively correlated with their levels of political participation across different types of Western democracies. 
We also extend previous research on dual screening by assessing whether and how the strength of this relationship varies across different individuals and contexts. With respect to individuals, we focus on the differential impact of dual screening among users with different levels of interest in politics. As regards the systemic context, we assess the extent to which the strength of Public Service Broadcasters (PSBs) moderates the relationship between dual screening and participation.

In addressing differential effects based on individual users' characteristics, we hope to contribute to solving an empirical puzzle. Some studies suggest dual screening might magnify political inequalities, while others imply quite the opposite.

Studies indicating dual screening may widen participatory gaps tend to treat it as a dependent variable. For instance, a qualitative analysis found that a key motivation for Italian Twitter users who dual screen political content is to perform a "full expression of citizenship: to act as informed publics and producers/distributors of information" (Selva 2016, p. 165). Another study characterized political dual screeners as an active minority of political junkies (Bentivegna and Marchetti 2015). Accordingly, Barnidge, Diehl, and Rojas (2018) found that Colombian citizens with higher socioeconomic status are more likely to dual screen for news, which may reinforce existing political inequalities based on resources. This line of scholarship focused on inequalities in who dual screens but does not directly investigate the differential effects of dual screening on participation, which may or may not result in further inequalities among those who dual screen. However, these studies suggest that citizens with higher level of political sophistication could be more likely to get satisfaction from dual screening politics and may be better equipped to manage its high cognitive load (Van Cauwenberge et al. 2014).

While these selection biases need to be taken seriously, other research suggests dual screening may mitigate rather than magnify participatory gaps by reaching less interested users with political content that impacts their participation. Freelon and Karpf (2015) 
highlight that irony and humor are amongst the most popular social media content generated during political TV events. Such messages can attract the attention of users who are less interested in politics by offering them relevant information that is easier and more rewarding to process, thus making the TV political experience more meaningful and potentially mobilizing. Dual screening may also encourage consumption of political broadcasts that users had not intended to watch. Vaccari and colleagues show that many users tune in to televised political debates after casually encountering social media posts about them (Vaccari et al. 2015, p. 1055).

More broadly, both Fletcher and Nielsen (2017) and Valeriani and Vaccari (2016) find that citizens who are relatively uninterested in the news and politics can stumble on political information on social media, and that as a result they receive a higher boost than highly interested users when it comes to the breadth of news sources they consume and their online political participation. Although these are general patterns related to exposure to news on social media rather than dual screening, they may be relevant for dual screening because it combines social media with television. Television news watchers are generally less politically engaged than political junkies on social media, and broadcast news may conceivably be an important gateway for politically uninvolved social media users to engage with political content and discussions, of the kinds they normally do not engage with online, while watching television. In this scenario, low-effort television news watching may lead to higher-effort political exchanges on social media. The reverse pattern - from social media to television-is also possible: citizens interacting with their acquaintances on social media may be exposed to political content they were not seeking out, learn about news via the comments they see online, and subsequently tune in to television to have a better sense of what is happening. Dual screeners who are scarcely interested in politics may be relatively more affected by exposure to political contents, as the marginal participatory utility of any new piece of 
information or any new opportunity for discussion should be higher for them than for those who are already highly interested.

To solve the empirical puzzle we have outlined, we aim to establish whether dual screening exacerbates or reduces political inequalities rooted in different levels of interest in politics - a key component of individuals' political involvement (Verba et al. 1995) and an attitude that is very stable throughout individuals' life cycles (Prior 2010). Since different studies suggest contradicting expectations, we formulate an open research question:

RQ1: Does interest in politics positively or negatively moderate the positive relationship between dual screening political content and participation?

Political dual screening does not happen in a contextual vacuum. Systemic characteristics that vary across countries influence the political content social media users are likely to see on television and, potentially, the practice of dual screening and the relationship between dual screening and participation. Assessing the role of cross-country differences requires comparative research designs, but studies addressing these issues in more than one country have been rare. In a 20-country comparison, Gil de Zúñiga and Liu (2017) found that political dual screening is more popular in media systems with less established markets for news and weaker freedom of the media. This suggests that the characteristics of media systems should be taken into consideration when assessing the relationship between dual screening and participation.

Research indicates that exposure to "hard" news about public affairs on television enhances citizens' political information, efficacy, and participation (De Vreese and Boomgaarden 2006; Aarts and Semetko 2003). Accordingly, we assess the extent to which dual screening's implications for participation vary as a function of political broadcasts in different media systems. We compare different countries where TV political content systemically differs, focusing on the strength of Public Service Broadcasters (PSBs) as a 
moderating systemic factor in the individual-level relationship between dual screening and participation.

The importance of PSBs for the quality of journalism, the vitality of the public sphere, and the health of democracy has been widely emphasized (Wahl-Jorgensen and Hanitzsch 2009). The strength and centrality of PSBs positively affect citizens' political knowledge (Curran et al. 2009; Soroka et al. 2012) and participation (Strömbäck and Shehata 2010). These effects of PSBs should extend to political television programming writ large in a country because strong PSBs can set the tone for broadcast journalism (Hallin and Mancini 2004).

Assessing the relationship between characteristics of the older and newer media systems requires theorizing how these two systems may interact with each other and with what effects. Anstead and Chadwick argue that "technologies can reshape institutions, but institutions will mediate eventual outcomes" (Anstead and Chadwick 2009, p. 58) and suggest that established media systems and political communication practices shape how digital media are integrated in parties' and citizens' toolkits. In thinking about how the implications of a newer digital practice such as dual screening may be shaped by older media institutions such as PSBs, we identify two theoretical configurations: adaptation and transformation.

With adaption we refer to the possibility that digital media reproduce, and possibly reinforce, patterns characterizing older media. According to this logic, dual screeners in media systems with strong PSBs should engage with high quality political broadcasts that enhance their activism and discussing those programs on social media should further magnify those participatory benefits. Conversely, dual screeners in media systems with weak PSBs should encounter broadcast content that is less beneficial to their participation, and the lower civic quality of those programs should also be reflected in discussions around them on social media, whose participants would reap lower participatory gains as a result. The strength of 
PSBs should therefore be mirrored by the strength of the association between dual screening and participation.

Transformation suggests the possibility that digital media counter-balance the impact of older media institutions. Accordingly, in media systems with weak PSBs, where political broadcasts are generally less informative and more politically biased, dual screening may yield higher participatory returns by filling the vacuum left open by the lower civic quality of televised content. For example, politically biased broadcasts may incentivize dual screeners to perform "corrective action" (Barnidge and Rojas 2014) by sharing verified and impartial information. Such actions may in turn increase the diversity and quality of political exchanges on social media vis-à-vis televised content. Conversely, when strong PSBs guarantee a generally high quality of political information that already enhances viewers' political knowledge and civic inclinations, any additional participatory boost that may result from dual screening may be comparatively smaller, as there is less room for dual screeners' participation to grow specifically as a result of dual screening. Under this scenario, we should expect an inverse relationship between the strength of PSBs and the strength of the relationship between dual screening and participation.

To be clear, adaptation and transformation should be seen as ideal-typical and fluid configurations, where many interdependent actors push and pull in different directions. They do not entail once-and-for-all solutions to the complex and evolving integration of established arrangements and disruptive technologies but suggest alternative pathways through which that integration may occur at a given time, in a given place, and for a given set of technologies and actors. To assess whether the logics of adaptation or transformation better capture the participatory implications of the integration between dual screening and PSBs, we ask this research question: 
RQ2: Does the strength of Public Service Broadcasters (PSBS) in different countries positively or negatively moderate the relationship between dual screening political content and participation?

\section{Data and Variables}

We employ surveys administered by Ipsos on panel-based online samples in Denmark, Germany, Greece, Italy, Poland, Spain, the United Kingdom, and the United States between June 2015 and December 2016, with an average participation rate of 20.5\%. Extensive information on our sample, measures, and alternative specifications of our models are available in the Online Appendix. ${ }^{1}$ We fielded the surveys immediately after a national general election in all countries apart from Germany and Italy, where they were administered outside of a national electoral period. We constructed samples $(N=2,500$ in the US, $N=1,750$ for the other countries) based on quotas that ensure representativeness of the adult population with internet access in each country by age, gender, region, occupational condition, and education.

Non-probability panel-based online samples like ours suffer from various limitations, whose statistical implications are difficult to address (Baker et al. 2010). If carefully constructed, however, they can correctly estimate relationships between political attitudes and behaviors (Pasek 2016), which is the focus of our study. Moreover, because online surveys are self-administered, responses are less affected by social desirability biases than face-to-face and telephone surveys (Kreuter et al. 2008). Finally, since only internet users can dual screen, our population of interest is the population with internet access, not the general population, so coverage bias should not be an issue.

Our dependent variable measures respondents' political participation online and offline. We constructed an index combining answers to questions probing respondents'

\footnotetext{
1 The Online Appendix is included among the Supplementary Materials to this article.
} 
engagement in six activities, four of which measured by a battery introduced by the question:

“People often carry out various activities in order to participate in politics. During the last 12

months, ${ }^{2}$ have you taken part in any of the activities listed below?" (Response modes were:

"Yes", “No", and "I don’t Remember". We included these items: "Distributing leaflets to

support a political or social cause", "Financing a party", "Participating in public rallies or

meetings on political issues", and "Contacting a political representative to support a cause".

We measured two other activities—signing petitions or referenda and trying to convince

others to vote for a party or candidate-by combining questions pertaining to online and

offline actions. For each activity, we coded as 1 respondents who answered they had

undertaken it either in general or exclusively online. ${ }^{3}$ Across the six items, Cronbach's $\alpha$ was

0.71, suggesting high consistency. The index of participation sums the number of activities

respondents performed, and thus ranges from 0-6. In discussing our findings on the direct

effects of dual screening (H1), we also discuss results of models predicting individual

activities (Figure 1 below).

Our main independent variable measures dual screening political content. To capture

the different broadcasts around which social media users dual screen, we asked a general

\footnotetext{
${ }^{2}$ The question asked in Germany and Italy referred to "six months" instead. In both countries, regional elections were held six months before our fieldwork started, involving seven regions in Italy and one in Germany. Our questions referred to the past six months to ensure that respondents' answers did not include the campaigning period for regional elections, which did not occur uniformly across the whole countries.

${ }^{3}$ As regards signing petitions, we combined answers to the item "Endorsing a petition or signing a referendum" from the main battery described above, with answers to the item "signed an online petition" included in a battery of activities conducted online, which was preceded by the question: "Various political activities are carried out via the Internet. During the past 12 months have you... ?"). As for trying to convince others to vote for a party or candidate, we combined answers to the item "Trying to convince someone to vote for a party, politician or political leader" from the main battery described above, with answers to the item "tried to convince someone to vote for a party leader, politician or party using email" included in our battery of web-based activities, and with answers to the item "tried to convince someone to vote for a specific party leader, politician or party" included in a battery of activities conducted on social media, introduced by the question: "Various political activities are carried out on social networks / social media platforms such as Facebook, Twitter, YouTube, etc. During the past 12 months have you... ?". Response modes were "Yes," "No," and "I don't Remember" for all batteries. When a respondent answered "Yes" to one of the questions we combined to measure an activity, we included them even if they answered "I don't Remember" to one of the others, because we had enough information to establish that the respondent had engaged in that activity. When respondents answered "No" to one question and "I don't Remember" to the other questions, we treated them as missing values, because we did not have enough information to establish that the respondent had or had not engaged in that activity.
} 
question ("Over the last two months, how often did you use social networks / social media platforms to read about and discuss televised news programs while aired?"). The question comprised three specific items, adapted from Gil de Zúñiga, Garcia-Perdomo, and McGregor (2015): “During news programs”, “During campaign speeches, interviews, or debates involving politicians", and "During news coverage of the elections". ${ }^{4}$ Response modes were: "Every day or almost every day", "A few times a week", “A few times a month", "Never or almost never", and "I don't remember". ${ }^{5}$ These items are highly consistent (Cronbach's $\alpha=$ 0.94). The variable used in our models is the sum of the answers to each item (ranging from 03), which results in a 0-9 scale. Table 1 shows descriptive statistics for the main dependent and independent variables and their constitutive components.

\section{Table 1 about here}

RQ1 and RQ2 address whether and how interest in politics and the strength of PSBs moderate the relationship between dual screening and participation. We measured interest in politics by asking: "In general, how interested are you in politics?". Response modes were: "Not at all interested", "Slightly interested", "Moderately interested", "Very interested”, and "I don't know". As regards the strength of each country's PSB, we followed Hallin and Mancini (2004) and Brüggemann et al. (2014) and divided our eight countries into three groups: Denmark, Germany, and the United Kingdom display strong PSBs; Greece, Italy, Poland, and Spain feature medium-strength PSBs; and the United States has a weak PSB. ${ }^{6}$ The model we employ

\footnotetext{
${ }^{4}$ In the German and Italian questionnaires, the last item was phrased as "During TV programs on political issues" to reflect the fact that no nationwide elections occurred before the surveys were fielded.

5 This question, as well as others relating to social media experiences and behaviors, were only asked to social media users in our sample (92.1\% of respondents). To avoid biases resulting from individuals' selecting themselves into using social media, we have also included non-users in our analyses, assigning them a value of 0 in the variables derived from questions on social media uses or experiences.

${ }^{6}$ Brüggeman and colleagues (2014: 1051) classified public broadcasting based on market share and public revenues. On this dimension, they ranked Denmark first, Germany second, and the UK third out of 17 countries.
} 
to answer RQ2 clusters respondents according to the group they belong to, treating strong PSBs as reference category.

We analyze the data with negative binomial regression, which is appropriate for modeling overdispersed count variables, as is the case for the index of political participation. ${ }^{7}$ We analyzed a pooled dataset comprising all eight countries, clustering standard errors by country to account for the fact that observations from the same country may be correlated in ways that are not captured by our models (Smets 2016). All models control for variables that previous research suggests affect the relationships of interest: gender, age, education, income, political efficacy, left-right self-placement, frequency of exposure to news via television and social media, and frequency of political talk on social media. Additional information on these variables is available in the Online Appendix.

Our dataset features missing values, generally resulting from the fact that some respondents chose to answer "I don't know" to one or more questions. Rather than introducing biases by limiting our analyses to respondents with no missing values (King et al. 2001), we performed multiple imputation using a chained equations method, which takes into account the different characteristics of the variables to be imputed. We also included a range of ancillary variables to employ as much information as possible when imputing missing values. Information on the multiple imputation procedure is available in the Online Appendix.

\footnotetext{
They ranked Spain $8^{\text {th }}$, Italy $11^{\text {th }}$, Greece $16^{\text {th }}$, and the United States $17^{\text {th }}$. The distance between Greece and the US, however, was much larger (1.65 in standardized scores) than that between Greece and Italy (.86) and between Greece and Spain (1.34). Qualitatively, there is a difference between Greece, where the PSB has historically had a strong presence (although it was closed between 2013-5) and the United States, where PSBs have always been residual in both funding and audience. We classified Poland, which is not covered by Hallin and Mancini (2004) and Brüggemann et al. (2014), as a medium-strength PSB because, while it controls a large share of the TV audience, commercial competitors play a substantial role and most of the PSB's revenues come from commercial sources (Dobek-Ostrowska 2012: 32-3).

${ }^{7}$ The modal category in the index is 0 (39.06\% of cases), followed by 1 (26.29\%) and 2 (16.39\%). A plot representing the distribution of the variable is provided in Figure A1 in the Online Appendix. Table A7 in the Online Appendix shows results of models that use Poisson regression, which are fully consistent with those reported here.
} 


\section{Findings}

Table 2 presents negative binomial regressions that predict the values of the index of political participation as a function of frequency of dual screening political content, interest in politics, strength of PSB in the respondents' country, interaction terms between these variables (Models 2 and 3), and relevant covariates.

\section{Table 2 about here}

Model 1 confirms that the direct relationship between dual screening political content and participation is positive and significant, as predicted by H1. The more frequently respondents dual screen, the more they participate. The effect sizes are substantial: the predicted value of the index of participation-which in the imputed dataset has a mean of 1.33 and a standard deviation of 1.5-rises substantially for average respondents who score at incrementally higher levels of the dual screening index: from 0.94 for non-dual screeners (score 0 ) to 1.13 for those who score 3 , to 1.35 for those who score 6 , to 1.61 for those who score the highest value (9). All these differences are statistically significant. To ensure this finding is not an artifact of the large sample size of the pooled dataset, we ran the same models on each country's data and obtained positive and significant coefficients in all eight countries. ${ }^{8}$

As discussed earlier, our dependent variable combines different modes of political participation, some of which occur entirely offline, some mainly online, and some across both domains. Moreover, the six activities require different types and amounts of resources. To better understand the relationship between dual screening and particular modes of political action, we estimated logistic regression models predicting each individual activity. The full

\footnotetext{
8 The B coefficients were (standard errors in parenthesis): Denmark 0.035 (0.009), Germany $0.079(0.014)$, Greece 0.028 (0.007), Italy 0.061 (0.008), Poland 0.062 (0.008), Spain 0.060 (0.008), UK 0.085 (0.010), USA 0.057 (0.007). In all countries, the coefficients were significant at $99.9 \%$ confidence levels.
} 
models are available in the Online Appendix. The coefficients for dual screening are all positive and significant at the $99 \%$ threshold. Figure 1 shows the effect size estimates for lowest (0), moderately low (3), moderately high (6) and highest (9) values of dual screening.

\section{Figure 1 about here}

Let us consider two hypothetical respondents who dual screen with different intensities (values of 3 and 6 in a 0-9 scale, roughly one standard deviation in our sample) while having average values in all other variables. The models predict the probability that these two respondents engage in leafleting should be nearly twice as large for the more frequent than the less frequent dual screener (10.6\% versus 5.6\%, a 90\% increase). The second largest predicted difference involves financing a party ( $8.9 \%$ versus $5.6 \%$, a $58 \%$ increase), followed by participating in rallies (15.5\% versus $11.1 \%$, a $39 \%$ increase), contacting a representative (15.5\% versus $12.2 \%$, a $27 \%$ increase), trying to convince someone to vote for a party or candidate ( $45.3 \%$ to $36.8 \%$, a $23 \%$ increase), and signing a petition ( $47.9 \%$ to $46.1 \%$, a $4 \%$ increase). As shown by the confidence intervals plotted in Figure 1, these differences are statistically significant for leafleting and participating in rallies, but not for the other activities. If we compare respondents whose dual screening frequency we scored as 0 versus 6 (or 3 versus 9), the differences are significant for all activities apart from contacting politicians and signing petitions. With respect to contacting, the difference between the lowest and highest levels of dual screening is significant, while this is not the case for signing petitions - even though the overall coefficient for dual screening in the regression is significant (see Table A8 in the Online Appendix).

RQ1 investigates whether the strength of the positive relationship between dual screening and participation decreases or increases as interest in politics grows, and our 
findings show that it decreases (Model 2 in Table 2). First, the coefficient for dual screening, which in this model captures the relationship between dual screening and participation among respondents not interested in politics, increases substantially compared to Model 1. Thus, the relationship between dual screening and participation is stronger among respondents with the lowest levels of political interest. Secondly, the interaction term between dual screening and interest in politics is negative and significant, which indicates that the strength of the positive relationship between dual screening and participation decreases when interest in politics increases. The effect size estimates plotted in Figure 2 show that, among average respondents who never (value of 0 ) and rarely (value of 3 ) dual screen, the predicted difference in participation between the most and least politically interested is significant at the $95 \%$ threshold. However, among respondents who dual screen political content more frequently (values of 6 and 9), the predicted levels of participation for respondents with high and low interest in politics are statically indistinguishable.

\section{Figure 2 about here}

Finally, Model 3 in Table 2 allows us to compare the strength of the association between dual screening and participation across countries where PSBs are strong, medium, and weak (RQ2). The country group with strong PSBs serves as the reference category, so the coefficients for the other two groups capture the differences with the group featuring Denmark, Germany, and the UK. The coefficient for dual screening, in turn, represents the relationship between dual screening and participation in countries with strong PSBs. The fact that such coefficient in Model 3 is higher than the coefficient in Model 1 suggests the correlation between dual screening and participation is stronger among respondents from strong-PSB countries than in the whole sample. Moreover, the negative and significant 
coefficients for the variables identifying countries with medium (Greece, Italy, Poland, and Spain) and weak (United States) PSBs suggests that the positive correlation between dual screening and participation is stronger where PSBs are strong. If we compare the predicted effect sizes from Model 3 between average respondents with the lowest and highest levels of dual screening, the value of the index of participation grows from 0.834 to $1.877(+125 \%)$ in countries with strong PSBs; from 0.967 to $1.510(+56 \%)$ in countries with medium PSBs; and from 1.104 to $1.672(+51 \%)$ in the United States (see Figure 3). Thus, the key difference is between countries with strong PSBs and all other countries, as the model coefficients and predicted values of participation are very similar between countries with medium and weak PSBs.

\section{Figure 3 about here}

\section{Limitations}

Our analysis has illuminated the relationship between dual screening political content and political participation and the way in which both individual factors - interest in politics-and systemic characteristics — strength of Public Service Broadcasting-moderate this relationship. However, we must acknowledge some limitations of our contribution.

First, because we rely on cross-sectional surveys, we cannot make any causal claims on the relationships we investigate, and we cannot rule out that some endogeneity may affect our findings. Dual screening may boost participation, but participation may also prompt individuals to dual screen. Only experimental designs can disentangle these associations, however difficult it seems to realistically administer dual screening as treatment. So far, experimental research on the political implications of dual screening has mostly addressed attitudinal rather than behavioral effects (Cameron and Geidner 2014). 
Secondly, we observed our variables of interest indirectly, through respondents' selfreports, rather than by unobtrusively collecting data on their online behavior. Therefore, some of our measures of political activity and exposure to information on social media are likely to be imperfect (Guess 2015). In principle, it is possible to collect social media data to gauge whether, how, and with whom some individuals discuss political broadcasts. By focusing on a specific program or campaign event, researchers can triangulate digital trace data with specific features of the broadcast (Shah et al. 2016) or track users' subsequent comments on social media (Vergeer and Franses 2016). However, it would be unrealistic to rely solely on unobtrusive online data to measure political behaviors occurring offline (such as attending a rally) or taking place on internet platforms that cannot be accessed by researchers due to privacy concerns (such as sending emails with political content), or to reliably measure political attitudes such as interest in politics and political efficacy. Albeit imperfect, self-reports help us gauge attitudes and behaviors that publicly observable online behaviors cannot reveal.

Thirdly, instead of differentiating between social media platforms, our questions and analysis referred generically to "social media and social networking platforms". This is a simplification, as digital platforms vary in their affordances and social uses, which may encourage different types of dual screening with specific participatory implications. Moreover, in assessing country variations, we employed clustered standard errors to reduce bias in their estimation, but we could not rely on multilevel modeling with countries as grouping variables - a more robust statistical strategy—as our dataset included only eight countries. Simulations suggest that at least 25 countries may be needed to obtain reliable estimates in multilevel models (Bryan and Jenkins 2016).

Finally, while our comparative research design and analysis were based on parsimonious yet solid theoretical grounds, we cannot test alternative theories that may 
explain the cross-country patterns we found. We selected eight Western democracies that differ-among other things - in the strength of their PSBs while being similar enough in others-such as the presence of a stable democratic regime and comparable levels of economic and technological development—-that we could assess whether the strength of PSBs moderates the relationship between dual screening and participation. In principle, however, we could have asked other questions focusing on different aspects of the media or the political system, but we could not simultaneously answer all these questions due to the relatively small number of countries we study. ${ }^{9}$ To overcome this limitation, scholars would need to simultaneously test alternative theories on the role of different systemic factors, which would require including more, and more diverse, countries than the eight covered here. This is the classic dilemma of "many variables, small number of cases" (Lijphart 1971, p. 685) in comparative research.

\section{Conclusions}

Our findings have substantive implications for political communication, media policy, and citizenship in contemporary democracies, as well as raising compelling normative questions. First, political content on television and discussion of it on social media can function as mutually reinforcing catalysts of citizens' political participation. Dual screening is an emerging practice where television and social media come together, mixing active and passive modes of engagement, derived from and enabled by both technologies. This combination can be beneficial for citizens' participation in politics. While previous research showed this to be the

\footnotetext{
${ }^{9}$ For illustrative purposes, the patterns in Figure 3 may be explained by differences in political parallelism on television, that is, the degree to which different media are tied to specific political parties or ideologies (Hallin and Mancini 2004). Television in Denmark, Germany, and the United Kingdom is characterized by lower levels of parallelism than in Greece, Italy, Poland, and Spain, while parallelism has arguably grown from low to medium in the United States. However, we do not see as clear a theoretical rationale for why this may be the case-let alone why one should set out studying this relationship-as we do for strength of PSBs. The reasons why we investigated whether cross-country differences in our main relationship of interest are rooted in strength of PSBs, as opposed to any other systemic factors, are entirely theoretical.
} 
case in individual countries, we have confirmed this positive relationship across eight diverse Western democracies. Moreover, we have shown that the participatory benefits of dual screening are relatively higher when it comes to more resource-intensive activities conducted in physical spaces and involving financial resources, while being proportionally smaller, though still noticeable, for lower-threshold activities that can be conducted mainly or entirely online. Although dual screening does not require particularly large amounts of resources, its implications for participation are by no means restricted to low-cost online activism and spill over into more demanding face-to-face action.

Secondly, dual screening can help close the participation gap between citizens with high and low levels of interest in politics. This is important since interest in politics is a crucial (Verba et al. 1995) and stable (Prior 2010) predictor of participation. As well as helping the already engaged become more active, dual screening disproportionately benefits less interested citizens. We argued this is because political exchanges on social media result in a greater marginal participatory utility for less involved citizens than for highly involved ones. When one cares less about politics, even a small nudge can make a difference, whereas when one is already involved, there is less room to grow. While political involvement is only one of the three main reasons-together with resources and mobilization networks (Verba et al. 1995) - why people participate in politics, by encouraging politically uninterested users to make their voice heard, dual screening can mitigate the impact of an important source of political inequality.

Thirdly, to the extent that the main social function and political justification of Public Service Broadcasters is to inform, educate, and entertain the public, dual screening is helping achieve that mission. Our findings suggest a new rationale for investing in robust public service media even as their role is increasingly questioned in Western democracies. Such investments, however, are more likely to pay off if PSBs are central in a country's 
broadcasting system and if they engage their audiences on social media, integrating their digital and broadcast activities to maximize the participatory benefits of dual screening. We also found dual screening adapts to, rather than transforming, established structures in a country's media system. Understanding digitally enabled hybrid practices such as dual screening requires situating them as part of the broader political communication ecosystem.

A final point concerns the normative foundations based on which we should assess the relationship between political communication-including dual screening-and participation. In the past two decades, digital media have often been touted as a solution to the problem of declining political participation in Western democracies. However, it is now clear that not all information circulating on social media is accurate, and some research suggests dual screening may even impair political knowledge (Gottfried et al. 2016). Assessing the implications of our study requires considering the possibility that some of the information voters exchange while dual screening may not be accurate, even as it may benefit participation.

An example helps clarify the normative challenges around dual screening during highstakes televised politics. In the US 2016 Presidential election, the public overwhelming saw Hillary Clinton as the winner of the first televised debate with Donald Trump-by $61 \%$ to $27 \%$, one of the largest margins in polling history (Gallup, 2016). Yet, the morning after the debate, "\#trumpwon" was the top worldwide trending hashtag on Twitter, as Trump himself proudly proclaimed in a tweet that further contributed to the spread of the meme. Analysis of the hashtag's propagation suggest small but cohesive networks of Trump supporters coordinated to share it (Lotan 2016). (It is unclear how many of the accounts participating in this concerted effort were automated and operated by foreign actors.) Thus, an active but small minority of users engaged in post-debate dual screening to exploit a relatively open digital platform and acquire favorable media coverage for Trump, as news media outlets duly reported this puzzling situation. Moreover, the Trump campaign bought banner 
advertisements on the websites of major news outlets announcing Trump had won the debate, which ended up appearing next to headlines of news reports of the same event (Rocheleau 2016). In all likelihood, these advertisements aimed to reassure Trump's supporters and motivate them to spread the word. While social media users and campaigns have every right to claim their candidate won a debate, this orchestrated effort based on serial tweets and advertisements spread an inaccurate impression-at least if we believe public opinion polls on representative samples of the population are more reliable to gauge public opinion than aggregation of social media content posted by supporters. Since evaluations of debate outcomes shape journalists' and viewers' assessments of the candidates, these dual screening practices raise important normative questions.

If political participation is enhanced by social media content-including content exchanged while dual screening - that persuasively conveys inaccurate information, citizens may provide input into the political system based on false premises and in support of policies and candidates on which they do not hold accurate beliefs (Flynn, Nyhan, and Reifler 2017). Because, as we have argued here, dual screening matters for political participation, we should be particularly concerned with the democratic quality of its content, the conduct of its main actors, and the health of its environment. 


\section{References}

Aarts, Kees, and Holli A. Semetko. 2003. "The divided electorate: Media use and political involvement." Journal of Politics 65 (3): 759-784.

Anstead, Nick, and Ben O’Loughlin. 2011. "The emerging viewertariat and BBC question time: Television debate and real-time commenting online." International Journal of press/politics 16 (4): 440-462.

Anstead, Nick, and Andrew Chadwick. 2008. "Parties, election campaigning, and the Internet Toward a comparative institutional approach." In The Routledge handbook of Internet politics, eds. Andrew Chadwick and Philip N. Howard. Abingdon, UK: Routledge.

Auverset, Lauren A., and Andrew C. Billings. 2016. "Relationships between social TV and enjoyment: A content analysis of The Walking Dead's story sync experience." Social Media+ Society 2 (3), 2056305116662170.

Baker, Reg, et al. 2010. "Research synthesis AAPOR report on online panels." Public Opinion Quarterly 74 (4): 711-781.

Barnidge, Matthew, Trevor Diehl, and Hernando Rojas. 2018. “Second Screening for News and Digital Divides." Social Science Computer Review 0894439317750840.

Barnidge, Matthew, Homero Gil de Zúñiga, and Trevor Diehl. 2017. “Second screening and political persuasion on social media." Journal of Broadcasting \& Electronic Media 61 (2): 309-331.

Barnidge, Matthew and Hernando Rojas. 2014. "Hostile media perceptions, presumed media influence, and political talk: Expanding the corrective action hypothesis." International Journal of Public Opinion Research 26 (2): 135-156.

Bentivegna, Sara, and Rita Marchetti. 2015. "Live tweeting a political debate: The case of the 'Italia bene comune'.” European Journal of Communication 30 (6): 631-647. 
Boulianne, Shelley 2015. “Social media use and participation: A meta-analysis of current research." Information, Communication \& Society 18 (5): 524-538.

Brüggemann, Michael, Sven Engesser, Florin Büchel, Edda Humprecht, and Laia Castro. 2014. "Hallin and Mancini revisited: Four empirical types of western media systems." Journal of Communication 64 (6): 1037-1065.

Bryan, Mark L and Stephen P. Jenkins. 2015. Multilevel modelling of country effects: A cautionary tale. European Sociological Review 32 (1): 3-22.

Cameron, Jaclyn, and Nick Geidner. 2014. “Something old, something new, something borrowed from something blue: Experiments on dual viewing TV and Twitter." Journal of Broadcasting \& Electronic Media 58 (3): 400-419.

Cantijoch, Marta, David Cutts, and Rachel Gibson. 2015. "Moving Slowly up the Ladder of Political Engagement: A 'Spill-Over' Model of Internet Participation." British Journal of Politics and International Relations 18 (1): 26-48.

Chadwick, Andrew. 2017. The Hybrid Media System: Politics and Power. Oxford: Oxford University Press, $2^{\text {nd }}$ edition.

Chadwick, Andrew, Ben O’Loughlin, and Cristian Vaccari. 2017. “Why People DualScreen Political Debates and Why It Matters for Democratic Engagement." Journal of Broadcasting and Electronic Media 61 (2): 220-239.

Choi, Boreum, and Yoonhyuk Jung. 2016. "The effects of second-screen viewing and the goal congruency of supplementary content on user perceptions." Computers in Human Behavior 64: 347-354.

Curran, James, Shanto Iyengar, Anker Brink Lund, and Inka Salovaara-Moring. 2009. "Media system, public knowledge and democracy: A comparative study." European Journal of Communication 24 (1): 5-26. 
De Vreese, Claes H., and Hajo Boomgaarden. 2006. "News, political knowledge and participation: The differential effects of news media exposure on political knowledge and participation." Acta Politica 41 (4): 317-341.

Dobek-Ostrowska, Boguslawa 2012. "Italianization (or Mediterraneanization) of the Polish media system." In Comparing media systems beyond the Western world, eds. Daniel Hallin, and Paolo Mancini. Oxford: Oxford University Press.

Eveland, William P. 2004. "The effect of political discussion in producing informed citizens: The roles of information, motivation, and elaboration." Political Communication 21 (2): 177-193.

Fletcher, Richard and Rasmus Kleis Nielsen. 2017. “Are people incidentally exposed to news on social media? A comparative analysis." New Media \& Society, 1461444817724170.

Flynn, D.J.; Brendan Nyhan, and Jason Reifler. 2017. "The nature and origins of misperceptions: Understanding false and unsupported beliefs about politics." Political Psychology 38 (S1): 127-150.

Freelon, Deen, and David Karpf. 2015. “Of big birds and bayonets: Hybrid Twitter interactivity in the 2012 presidential debates." Information, Communication \& Society 18 (4): 390-406.

Gallup. 2016. “Clinton's Victory on the Larger Side for Modern Debates”. URL (accessed 20 March 2018): http://news.gallup.com/poll/195923/clinton-debate-victory-larger-sidemodern-debates.aspx

Giglietto, Fabio, and Donatella Selva. 2014. "Second screen and participation: A content analysis on a full season dataset of tweets." Journal of Communication 64 (2): 260-277.

Gil de Zúñiga, Homero, Victor Garcia-Perdomo, and Shannon C. McGregor. 2015. "What is second screening? Exploring motivations of second screen use and its effect on online political participation." Journal of Communication 65 (5): 793-815. 
Gil de Zúñiga, Homero, Logan Molyneux, and Pei Zheng. 2014. “Social media, political expression, and political participation: Panel analysis of lagged and concurrent relationships." Journal of Communication 64 (4): 612-634.

Gil de Zúñiga, Homero, and James H. Liu. 2017. “Second Screening Politics in the Social Media Sphere: Advancing Research on Dual Screen Use in Political Communication with Evidence from 20 countries." Journal of Broadcasting and Electronic Media 61 (2): 193-219.

Gottfried, Jeffery A., Bruce W. Hardy, Lance R. Holbert, Kenneth M. Winneg, and Kathleen Hall Jamieson. 2016. “The Changing Nature of Political Debate Consumption: Social Media, Multitasking, and Knowledge Acquisition." Political Communication 34 (2): 172-199.

Guess, Andrew M. 2015. “Measure for Measure: An Experimental Test of Online Political Media Exposure." Political Analysis 23 (1): 59-75.

Hallin, Daniel C., and Paolo Mancini. 2004. Comparing media systems: Three models of media and politics. Cambridge: Cambridge university press.

King, Gary, James Honaker, Anne Joseph, and Kenneth Scheve. 2001. “Analyzing incomplete political science data: An alternative algorithm for multiple imputation." American political science review 95 (1): 49-69.

Kreiss, Daniel. 2016. “Seizing the moment: The presidential campaigns' use of Twitter during the 2012 electoral cycle." New Media \& Society 18 (8): 1473-1490.

Kreuter, Frauke, Stanley Presser, and Roger Tourangeau. 2008. “Social desirability bias in CATI, IVR, and Web surveys the effects of mode and question sensitivity." Public Opinion Quarterly 72 (5): 847-865.

Lijphart, Arend. 1971. "Comparative politics and the comparative method." American Political Science Review 65 (03): 682-693.

Lotan, Gilad. 2016. “No, Russians did not start the \#TrumpWon debate meme. Here's what really happened." Washington Post, URL (accessed 20 March 2018): 
https://www.washingtonpost.com/news/monkey-cage/wp/2016/10/03/no-russians-didnot-start-the-trumpwon-debate-meme-heres-what-reallyhappened/?utm_term=.44393c8cb7b3

McLeod, Jack M., Dietram A. Scheufele, and Patricia Moy. 1999. “Community, communication, and participation: The role of mass media and interpersonal discussion in local political participation." Political communication 16 (3): 315-336.

Norris, Pippa. 2000. A Virtuous Circle: Political Communications in Postindustrial Societies. Cambridge: Cambridge University Press.

Pasek, Josh. 2016. “When will nonprobability surveys mirror probability surveys? Considering types of inference and weighting strategies as criteria for correspondence." International Journal of Public Opinion Research 28 (2): 269-291.

Pew Research Center. 2016. "TV still the top source for election results, but digital platforms rise." URL: http://www.pewresearch.org/fact-tank/2016/11/21/tv-still-the-topsource-for-election-results-but-digital-platforms-rise/ (accessed 31 March 2017).

Prior, Markus 2010. “You've either got it or you don't? The stability of political interest over the life cycle." The Journal of Politics 72 (3): 747-766.

Putnam, Robert D. 1995. “Tuning in, tuning out: The strange disappearance of social capital in America." PS: Political science \& politics 28 (04): 664-683.

Reuters Institute for the Study of Journalism (2016). Reuters Digital News Report 2016. Available at http://www.digitalnewsreport.org/ (accessed 31 March 2017).

Robinson, Michael J. 1976. "Public Affairs Television and the Growth of Political Malaise: The Case of" The Selling of the Pentagon"." The American Political Science Review 70 (2): 409-432. 
Rocheleau, Matt. 2016. "Why is \#TrumpWon trending on Twitter?" Boston Globe, URL (accessed 20 March 2018): https://www.bostonglobe.com/metro/2016/09/27/whytrumpwon-trending-twitter/HVvPNofMy73xZcTSPipZvO/story.html

Selva, Donatella. 2016. "Social television: Audience and political engagement." Television \& New Media 17 (2): 159-173.

Shah, Dhavan V., Alex Hanna, Erik P. Bucy, David S. Lassen, Jack Van Thomme, Kristen Bialik, JungHwan Yang, and Jon C. W. Pevehouse. 2016. “Dual screening during presidential debates: Political nonverbals and the volume and valence of online expression." American Behavioral Scientist 60 (14): 1816-1843.

Smets, Kaat. 2016. “Revisiting the political life-cycle model: Later maturation and turnout decline among young adults." European Political Science Review 8 (02): 225-249.

Soroka, Stuart, Blake Andrew, Toril Aalberg, Shanto Iyengar, James Curran, Sharon Coen, Kaori Hayashi, Paul Jones, Giampietro Mazzoleni, June Woong Rhee, and D. Rowe. 2013. “Auntie knows best? Public broadcasters and current affairs knowledge.” British Journal of Political Science 43 (4): 719-739.

Strömbäck, Jesper, and Adam Shehata. 2010. “Media malaise or a virtuous circle? Exploring the causal relationships between news media exposure, political news attention and political interest." European Journal of Political Research 49 (5): 575-597.

Trilling, Damian. 2015. "Two different debates? Investigating the relationship between a political debate on TV and simultaneous comments on Twitter." Social Science Computer Review 33 (3): 259-276.

Vaccari, Cristian, Andrew Chadwick, and Ben O'Loughlin. 2015. "Dual screening the political: Media events, social media, and citizen engagement." Journal of Communication 65 (6): 1041-1061. 
Valeriani, Augusto, and Cristian Vaccari. 2016. "Accidental exposure to politics on social media as online participation equalizer in Germany, Italy, and the United Kingdom." New Media \& Society 18 (9): 1857-1874.

Van Cauwenberge, Anna, Gabi Schaap, and Rob Van Roy. 2014. “"TV no longer commands our full attention": Effects of second-screen viewing and task relevance on cognitive load and learning from news." Computers in Human Behavior 38: 100-109.

Verba, Sidney, Kay L. Schlozman., and Henry E. Brady. 1995. Voice and equality: Civic voluntarism in American politics. Cambridge, MA: Harvard University Press.

Vergeer, Maurice, and Philip H. Franses. 2016. "Live audience responses to live televised election debates: time series analysis of issue salience and party salience on audience behavior." Information, Communication \& Society 19 (10): 1390-1410.

Yang, Hongwei C., and Jean L. DeHart. 2016. "Social media use and online political participation among college students during the US election 2012." Social Media+ Society 2 (1), 2056305115623802. 
Table 1 - Descriptive Statistics for the Index of Political Participation and Political Dual Screening , by Country

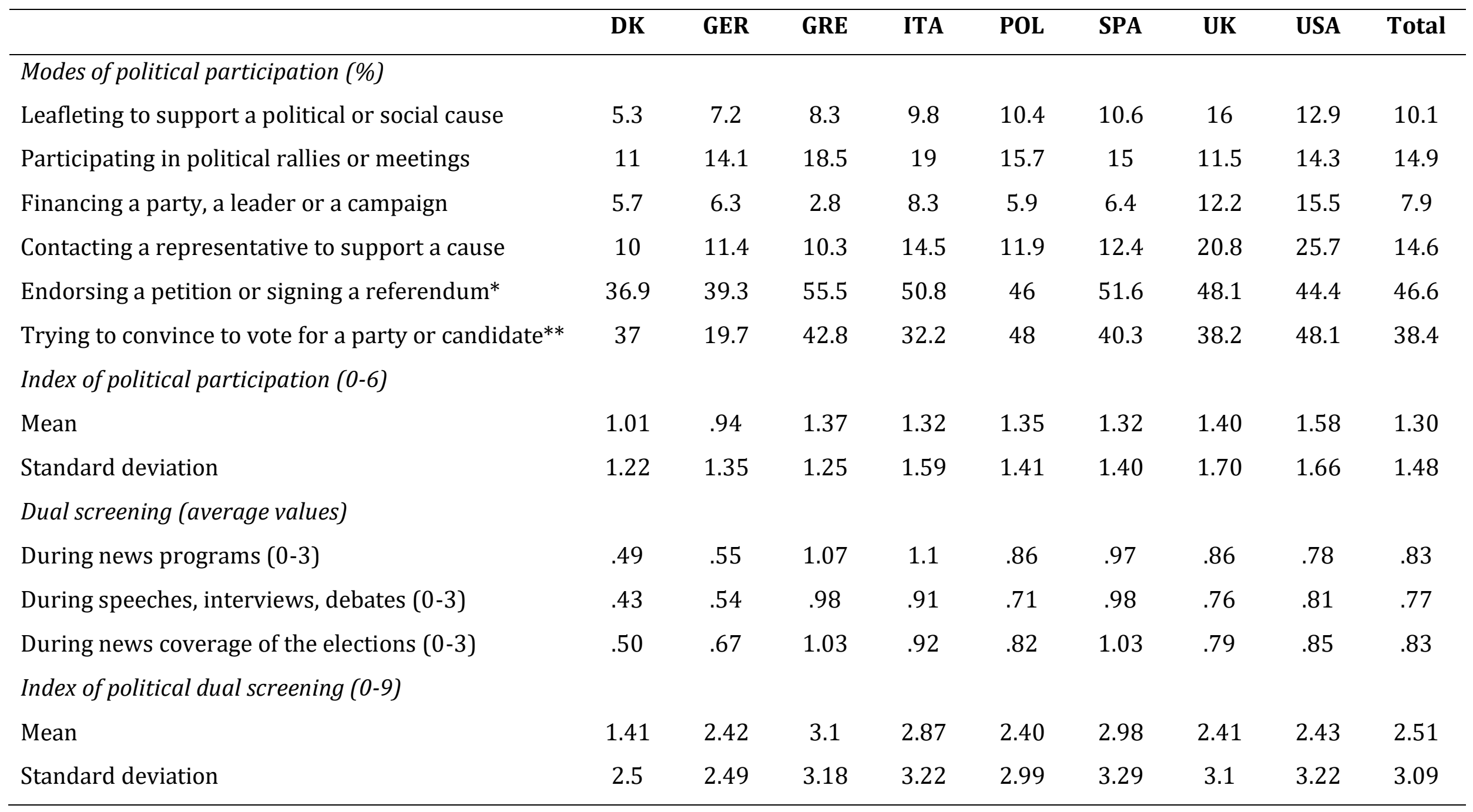

* Offline or online

** Offline, via email, or using social media 
Table 2 - Dependent Variable: Political Participation (0-6 Index)

\begin{tabular}{|c|c|c|c|c|c|c|}
\hline & \multicolumn{2}{|c|}{ Model 1} & \multicolumn{2}{|c|}{ Model 2} & \multicolumn{2}{|c|}{ Model 3} \\
\hline & B & s.e. & B & s.e. & B & s.e. \\
\hline Dual Screening political content & $0.059 * * *$ & 0.007 & $0.181^{* * *}$ & 0.015 & $0.090^{* * *}$ & 0.016 \\
\hline Interest in politics & $0.207^{* * *}$ & 0.044 & $0.394^{* * *}$ & 0.049 & $0.208^{* * *}$ & 0.042 \\
\hline Dual Screening * Interest in politics & & & $-0.053^{* * *}$ & 0.005 & & \\
\hline \multicolumn{7}{|c|}{ Strength of Public Service Broadcaster (High = ref.) } \\
\hline Medium PSB & & & & & 0.147 & 0.099 \\
\hline Weak PSB & & & & & $0.281^{* * *}$ & 0.079 \\
\hline Dual Screening * Medium PSB & & & & & $-0.041^{*}$ & 0.019 \\
\hline Dual Screening * Weak PSB & & & & & $-0.044^{* * *}$ & 0.014 \\
\hline Political news via TV & $-0.021^{*}$ & 0.010 & $-0.030^{* *}$ & 0.011 & -0.016 & 0.010 \\
\hline Political news via social media & $0.050 * *$ & 0.017 & $0.043^{*}$ & 0.017 & $0.045^{* *}$ & 0.015 \\
\hline Political talk on social media & $0.251^{* * *}$ & 0.018 & $0.258^{* * *}$ & 0.019 & $0.248^{* * *}$ & 0.018 \\
\hline \multicolumn{7}{|l|}{ Ideology (Unaligned = ref.) } \\
\hline Left & $0.390 * * *$ & 0.073 & $0.369 * * *$ & 0.065 & $0.390 * * *$ & 0.072 \\
\hline Center-left & $0.295^{* * *}$ & 0.063 & $0.271^{* * *}$ & 0.059 & $0.302^{* * *}$ & 0.064 \\
\hline Center & $0.189 * *$ & 0.060 & $0.163^{* *}$ & 0.055 & $0.189 * * *$ & 0.056 \\
\hline Center-right & $0.311^{* * *}$ & 0.091 & $0.293^{* * *}$ & 0.083 & $0.310^{* * *}$ & 0.084 \\
\hline Right & $0.395^{* * *}$ & 0.080 & $0.389 * * *$ & 0.073 & $0.384^{* * *}$ & 0.073 \\
\hline Political efficacy & $0.050^{* * *}$ & 0.009 & $0.043^{* * *}$ & 0.008 & $0.048^{* * *}$ & 0.009 \\
\hline Gender (male) & $0.088^{* * *}$ & 0.014 & $0.081^{* * *}$ & 0.015 & $0.087^{* * *}$ & 0.014 \\
\hline Age & 0.001 & 0.001 & 0.000 & 0.001 & 0.001 & 0.001 \\
\hline Education & 0.038 & 0.022 & 0.043 & 0.024 & 0.019 & 0.017 \\
\hline Income & 0.005 & 0.005 & 0.007 & 0.005 & 0.004 & 0.005 \\
\hline Constant & $-1.266^{* * *}$ & 0.123 & $0.081^{* * *}$ & 0.015 & $-1.356^{* * *}$ & 0.157 \\
\hline $\mathrm{F}$ & \multicolumn{2}{|c|}{390.16} & \multicolumn{2}{|c|}{504.41} & \multicolumn{2}{|c|}{16584.98} \\
\hline Prob $>$ F & \multicolumn{2}{|c|}{0.000} & \multicolumn{2}{|c|}{0.000} & \multicolumn{2}{|c|}{0.000} \\
\hline
\end{tabular}

Note. $N=14,750$ for all models.

Cell entries are unstandardized coefficients for negative binomial regressions, with robust standard errors clustered by country. Standard measures of goodness of fit are not reported because the models use multiply imputed data.

PSB $=$ Public Service Broadcaster.

${ }^{* * *} \mathrm{p} \leq 0.001{ }^{* *} \mathrm{p} \leq 0.01 * \mathrm{p} \leq 0.05$ 
Figure 1 - Effect Size Estimates of Six Modes of Political Participation as a Function of Different Frequencies of Dual Screening, with 95\% Confidence Intervals

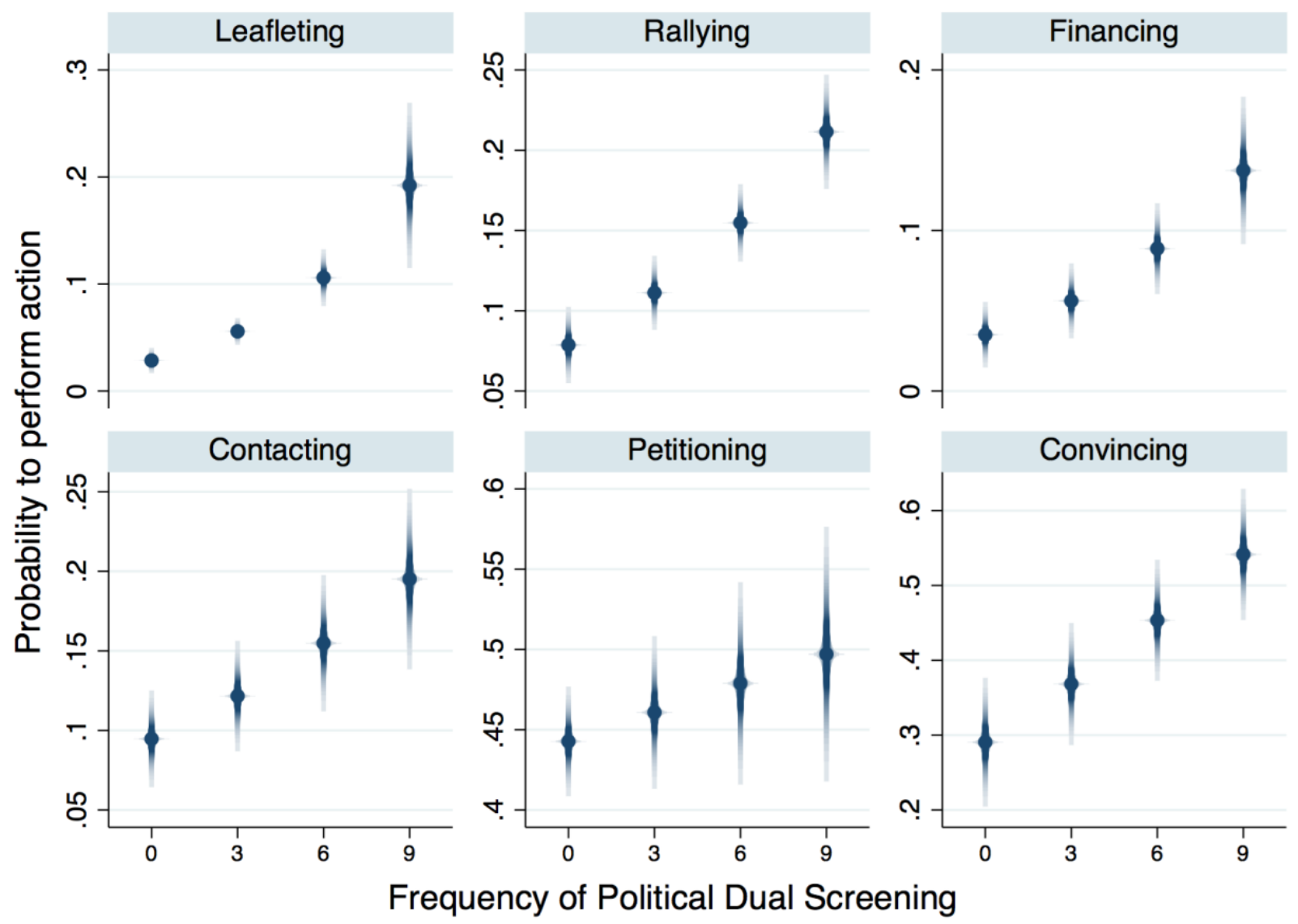

Note: the values are calculated based on the coefficients in Table A8 in the Online Appendix. The estimates are based on the means among respondents for all variables apart from political dual screening, estimated at values of $0,3,6$, and 9. The scales of the Y axis differ across each plot to accommodate the different ranges of variation in the predicted probability of each outcome. 
Figure 2 - Effect Size Estimates of Political Participation as a Function of Political Dual Screening and Interest in Politics, with 95\% Confidence Intervals

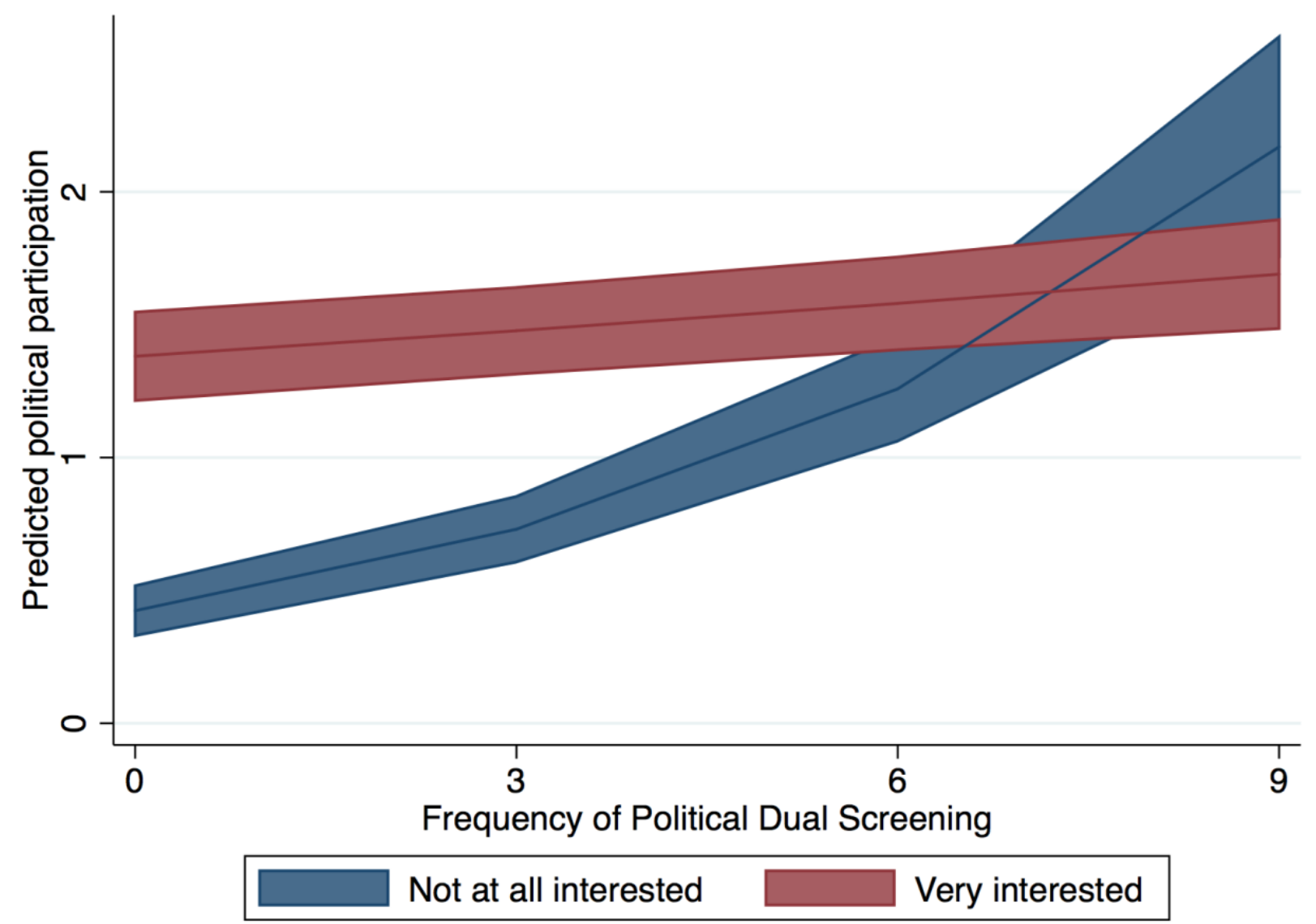

Note: the values are calculated based on the coefficients in Model 2 (Table 2). The estimates are based on the means among respondents for all variables apart from political dual screening (values of $0,3,6$, and 9) and interest in politics (values of 0 and 3). 
Figure 3 - Effect Size Estimates of Political Participation as a Function of Political Dual Screening in Countries with Strong, Medium, and Weak PSBs, with 95\% Confidence Intervals

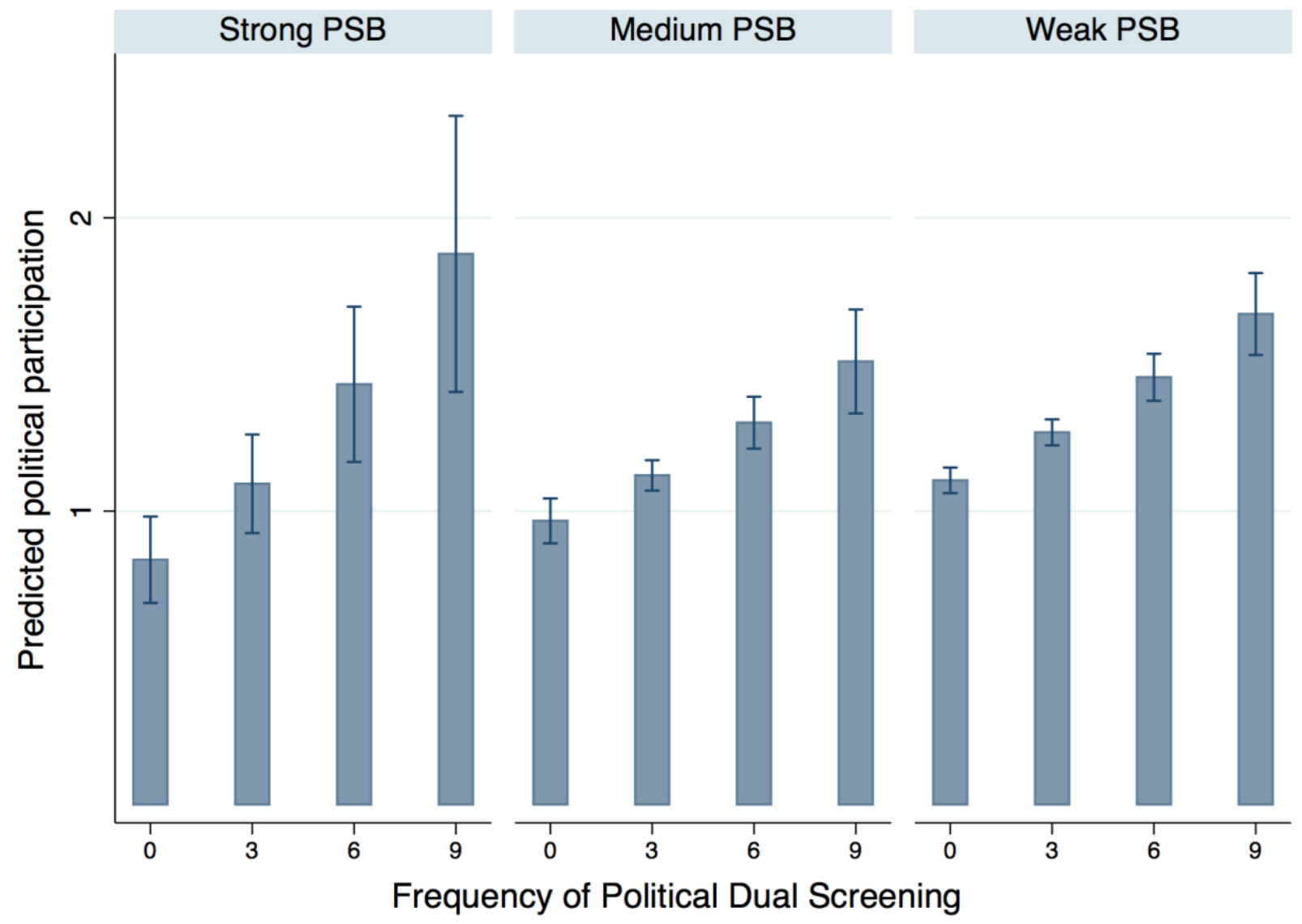

Note: the values are calculated based on the coefficients in Model 3 (Table 2). The estimates are based on the means among respondents for all variables apart from political dual screening (estimated at values of $0,3,6$, and 9). 